\title{
Capítulo i \\ El campo de la comunicación: algunas discusiones abiertas
}

\author{
Magdalena Sofía Paláu Cardona* \\ Instituto Tecnológico y de Estudios Superiores de Occidente
}

En este artículo se propone una nueva mirada en el acercamiento a cuatro aspectos centrales en la discusión abierta respecto del campo de la comunicación. Los aspectos abordados son: el estatuto disciplinar de los estudios de la comunicación, el enfoque para la construcción de objetos de estudio, las teorías y metodologías pertinentes para el estudio de la comunicación y las articulaciones posibles entre los subcampos de la comunicación. El supuesto que está a la base de esta lectura es que estos cuatro aspectos no se han debatido suficientemente y en forma incluyente aunque se plantean reiteradamente por los agentes del campo.

This article proposes a fresh reading of four aspects which are central to the ongoing discussion concerning the field of communication: the disciplinary statute of communication studies, approaches to the construction of study objects, pertinent theoretical and methodological frameworks for the study of communication, and possibilities of articulation among the sub-fields of communication studies. Underlying this reading is the assumption that, though repeatedly brought up and discussed by field actors, these four aspects have been debated neither sufficiently nor in relation to one another.

* Profesora del Departamento de Estudios Socioculturales del ITESO. Licenciada en Ciencias de la Comunicación por el ITESO, Maestra en Ciencias Sociales por la Universidad de Guadalajara. Actualmente estudia el Doctorado en Estudios Científico - Sociales, línea de Comunicación, cultura y sociedad en el ITESO. Correo electrónico: spalau@iteso.mx 


\section{INTRODUCCIÓN}

Aproximarse o reaproximarse al campo de la comunicación es una oportunidad de repensarlo y, al repensarlo, construir nuevos significados y nuevas interpretaciones sobre él. Los significados e interpretaciones que se construyen tienen el propósito general de aportar nuevos elementos a una discusión iniciada hace ya muchos años. Sin desconocer la riqueza y diversidad de la trayectoria del campo de la comunicación en nuestras sociedades contemporáneas, propongo aquí cuatro aspectos que, desde mi punto de vista, continúan siendo debates abiertos en el campo de la comunicación.

En el primer apartado se presenta una discusión que coloca en el centro el estatus disciplinar de los estudios de la comunicación. La discusión intenta contextualizar, en un primer momento, la emergencia del campo como campo académico en el pensamiento de las humanidades y las ciencias sociales y en sus respectivos procesos de institucionalización y disciplinarización. Desde este contexto se reconocen los movimientos que han seguido los estudios de la comunicación, desde su intento por colocarse como una disciplina científica, pasando por la explosión y fragmentación de posibles objetos, hasta la propuesta de asignarle un estatus transdisciplinario y concebirlo como un campo.

En el segundo apartado se desarrolla con más detalle una discusión que ha acompañado, desde hace muchos años, al campo académico de la comunicación. La disyuntiva entre estudiar la comunicación desde los medios masivos o estudiarla desde la cultura. Aunque para algunos esta discusión ha sido supera$\mathrm{da}$, aún quedan elementos y agentes que parecieran buscar regresar al pasado. Otros consideran que es un "malentendido" a la propuesta del estudio de la comunicación desde la cultura a través de las mediaciones.

El tercer apartado intenta colocar algunas bases para la discusión respecto de los objetos y metodologías pertinentes a los estudios de comunicación. Recojo aquí dos aproximaciones teóricas a la comunicación que comparten la intención de construir una mirada que abarque la complejidad del fenómeno, es por ello que creo que pueden ser un buen punto de partida para la discusión. No se consideran éstas aproximaciones teóricas como las únicas vías de entrada a la discusión, sino simplemente como propuestas que pueden servir de punto de partida. Evidentemente hay muchas otras propuestas teóricas que han estado presentes en el trabajo de investigación de la comunicación.

El cuarto apartado recoge una antigua preocupación respecto de la desarticulación del campo de la comunicación. Es la invitación a repensar, a través 
de la reconstrucción del proceso de emergencia y conformación del campo, caminos posibles para la fertilización productiva entre los subcampos: académico (de formación profesional y de investigación) y profesional (con todas las variantes del ejercicio profesional existente, incluida la investigación comercial o aplicada).

Los temas aquí discutidos no son nuevos, tampoco se recogen, en este artículo, todas las discusiones que se han abierto en la historia del campo, sin embargo se recogen algunos que aparecen como centrales para el fortalecimiento del campo de la comunicación. Es el intento de una mirada nueva sobre temas ya instalados en la discusión. Es la propuesta de una relectura a partir del pensamiento de muchos otros que ha quedado impreso en las páginas de sus libros, artículos y conferencias. Es el esfuerzo por construir una nueva intertextualidad que permita una nueva discusión sobre estos asuntos. Es, finalmente, una invitación a repensar juntos algunos de los temas que nos van constituyendo como campo.

\section{ESTATUTO DISCIPLINARIO DE LOS ESTUDIOS DE COMUNICACIÓN}

\section{Contexto cientifico del surgimiento de estudios de la comunicación}

Los estudios de comunicación aparecieron en un momento histórico y científico particulares: se desarrollaron en el mundo moderno y al interior del sistema mundial de universidades. En este contexto predominaba el cientificismo, una corriente de pensamiento que sostiene que la ciencia es el único modo legítimo de conocimiento, que existe el mundo real y la posibilidad de conocerlo empíricamente, aunque sea parcialmente. Con la modernidad se desarrolló una estructura de conocimiento de "dos culturas" y un divorcio entre la ciencia y la filosofía. En el mundo moderno hubo un marcado aumento del prestigio cultural de la ciencia a expensas del decrecimiento del prestigio cultural de las humanidades y la filosofía.

Las ciencias sociales se constituyeron, a partir del Siglo XIX, "a la sombra de la dominación cultural de la ciencia newtoniana e internalizaron la lucha entre estas dos culturas." (Wallerstein, 2004, p.17) Las ciencias sociales estuvieron en medio de la lucha entre las ciencias naturales y las humanidades. Este es el contexto en el que se dio el proceso de disciplinarización de las ciencias sociales. En este proceso participaron cinco países (Inglaterra, Francia, Alemania, Italia y Estados Unidos) y se conformaron seis disciplinas: la historia, la economía, la ciencia política, la sociología, la antropología y los estudios orientales. Según 
Wallerstein (1996) esta división disciplinar respondió a tres ejes distintos. El eje presente/pasado, en donde la historia trabaja sobre el pasado y el resto de las disciplinas se enfocan en el presente. El segundo eje fue el del espacio hipotético en donde la economía se interesaba por el mercado, la ciencia política por el estado y la sociología en la sociedad civil. El tercer eje respondía a la división entre el mundo civilizado y los "otros". Las cuatro primeras más interesadas en el occidente, es decir, en lo que se consideraba como el mundo civilizado; mientras que la antropología y los estudios orientales se interesaron por los países no occidentales, los considerados "otros".

Los estudios de comunicación, como tales, surgieron con el advenimiento de los medios electrónicos de comunicación de masas, primero la radio y posteriormente la televisión. Si queremos remontarnos a los orígenes del pensamiento moderno sobre la comunicación es necesario entender que hay dos grandes núcleos de pensamiento de los que abreva la reflexión sobre comunicación: las humanidades y las ciencias sociales.

Desde las humanidades la reflexión sobre comunicación está muy ligada a la teoría del conocimiento que tienen, como base común, las preguntas respecto de la construcción del significado y el tema de la intersubjetividad, como lo señala Jensen: “¿Cómo y por cuáles significados es posible compartir la comprensión de ciertos significados?”. Con la aparición de los medios de comunicación la pregunta se amplía “¿Cuáles son los rasgos relativamente fijos en cada significado de comunicar y cómo estos rasgos hacen física, psicológica y socialmente diferentes unos medios de otros y de la interacción cara a cara? " (Jensen, 2002, p. 16)

Las tradiciones más importantes de la historia de las ideas y que han alimentado la reflexión en comunicación son: la retórica, la hermenéutica, la fenomenología y la semiótica, siendo esta última la que más ha aportado en la conformación de los estudios de medios como un campo específico.

Desde las ciencias sociales hay que considerar que la concepción de la sociedad como un organismo, el desarrollo industrial y la división del trabajo, en síntesis, el desarrollo del capitalismo, es lo que inspira las primeras ideas respecto de la comunicación. En estas concepciones primeras, la comunicación, por un lado, está muy relacionada a la idea de vías de comunicación para el flujo de bienes y por el otro, es vista como un factor de integración de ese organismo-sociedad. Estas dos concepciones son denominadas por Carey (1989) como la visión de "transmisión" y la visión "ritual" de la comunicación y han estado presentes desde el siglo XIX, antes de la aparición de los medios masivos de comunicación. 
Con el auge y crecimiento de los medios de comunicación masiva se desarrollaron también algunas teorías que intentan explicar este fenómeno social. Al mismo tiempo comienza a desarrollarse, dentro de las Ciencias Sociales, la disciplina de la sociología. Estos dos elementos intervinieron en los primeros desarrollos teóricos que, sobre la comunicación, surgieron en Estados Unidos y Europa.

Después de la Segunda Guerra Mundial la estructura de las ciencias sociales colapsó por diversas razones y comenzaron a expandirse los campos de estudio. El campo referido específicamente a la comunicación estaba en una etapa inicial de constitución y en el intento por allegarse el reconocimiento como una disciplina con límites bien definidos. Al colapsar la división disciplinaria de las ciencias sociales, los estudios de comunicación se enfrentaron a la disyuntiva de consolidarse como una disciplina específica o abrirse al reto de pensarse, junto al resto de las disciplinas de las ciencias sociales, de un modo nuevo.

\section{INTENTO POR CONSTITUIR UNA DISCIPLINA BIEN DELIMITADA}

Con lo anteriormente expuesto queda suficientemente claro que el campo de estudios de la comunicación, en la primera mitad del Siglo XX, siguió el movimiento generalizado de las ciencias sociales por conseguir un estatuto científico. Los esfuerzos de agentes del campo, como Wilbur Schram, por reunir un cuerpo de conocimientos teóricos, un grupo de objetos de estudio específicos y una orientación metodológica son clara evidencia de ese impulso por alcanzar el estatus disciplinar para los estudios de comunicación. En ese esfuerzo predominaron las metodologías con perspectiva cuantitativa, los medios de comunicación de masas como objeto de estudio y las teorías en relación con los efectos de la comunicación de masas.

Estos esfuerzos llegaron "tarde" en términos del desarrollo de las ciencias sociales, llegaron en el momento en el que la división disciplinaria de las ciencias sociales era fuertemente cuestionada y se imponía un movimiento generalizado hacia la interdisciplinariedad. Sobre este tema Renato Ortiz (1999) afirma que en las ciencias sociales "la especialización disciplinaria, subdisciplinaria y temática alcanzó un grado tal en el Siglo XX que comprometió la frontera de la ciencia social, devino en un movimiento de fragmentación que no permite que se formulen un conjunto de problemas que no se pueden formular desde una disciplina particular”. (Ortiz, 1999, p.30) Un segundo elemento que ha transformado la investigación en las ciencias sociales es la transformación del momento 
histórico mundial, "con la globalización diversos aspectos de la realidad social se ven penetrados por un conjunto de fuerzas que recomponen el marco de las relaciones sociales, por lo que es necesario dar paso al estudio de las relaciones sociales mundializadas" (Ortiz, 1999, p. 36), es decir, los objetos de estudio de las ciencias sociales se han transformado y por lo tanto las ciencias sociales habrán de cambiar también. El cuestionamiento a las ciencias sociales y sus consecuencias inmediatas afectaron el desarrollo de los estudios de comunicación.

LA INTER - POST - ANTI - DISCIPLINARIEDAD DE LOS ESTUDIOS

DE LA COMUNICACIÓN

Después de 1945, la innovación académica más importante fue la creación de estudios por áreas o regiones. Estos nuevos estudios por áreas eran, por definición, "multidisciplinarios". Las prácticas académicas conjuntas de científicos de diversas especialidades "dejaron ver lo mucho que hay de artificial en las rígidas divisiones institucionales de conocimiento asociado a las ciencias sociales" (Vasallo, 2001, p. 50)

Una manera de enfrentar el desdibujamiento de las líneas divisorias entre las disciplinas de las ciencias sociales y la heterogeneidad de las mismas al irse estirando los límites de sus objetos de estudio y debilitándose la coherencia de las premisas intelectuales que se habían esgrimido para defender una existencia separada, fue la creación de ámbitos interdisciplinarios. La interdisciplinariedad suponía la combinación de diversas disciplinas sobre un objeto, esto llevó a la conformación de un equipo multidisciplinario para el estudio de dicho objeto. Otra forma que tomó la interdisciplinariedad fue la de colocar un objeto en la frontera de dos o más disciplinas.

El resultado de este esfuerzo interdisciplinario fue un acercamiento importante entre las disciplinas existentes, pero no implicó "una fructífera fertilización recíproca entre las disciplinas" (Vasallo, 2001, p.51) Los estudios de comunicación operaron, en este esfuerzo y en algunos casos, como un ámbito interdisciplinario y, en otros, como un campo disciplinar particular que aportaba desde su especificidad a estudios en los que intervenían diversas disciplinas.

Ante el desdibujamiento de las líneas divisorias entre las disciplinas de las ciencias sociales, algunos académicos interesados en los estudios de comunicación recurrieron a otras dos vías. La primera fue considerar los estudios de comunicación como un ámbito post-disciplinario, es decir más allá y posterior a la disciplinarización de las ciencias sociales. Los estudios de comunicación se 
presentaron como una alternativa que proponía objetos de estudio incorporando las perspectivas de múltiples disciplinas simultáneamente. Este esfuerzo no fructificó pues los agentes no contaban con suficiente peso específico y relativo en la lucha al interior del campo científico.

Una segunda vía, tomada por un grupo minoritario de académicos, fue la antidisciplina. Esta posición implica un rompimiento más radical, no sólo con la forma en que se estructuró el campo de las ciencias sociales en términos institucionales y disciplinares, sino como un cuestionamiento al pensamiento científico ortodoxo como única forma de conocimiento válido. Esta vía antidisciplinaria se afinca en "la dificultad actual que experimenta la ciencia, el descrédito a partir de la crítica respecto de su posible subjetividad y el cuestionamiento a su desinterés y a su pretendido carácter extrasocial." (Wallerstein, 2004, p. 13) Adicionalmente, implica la conciencia de que la realidad del mundo es cambiante y por lo tanto las interpretaciones que de él se hacen serán siempre transitorias.

\section{LA TRANSDISCIPLINARIEDAD DE LOS ESTUDIOS DE COMUNICACIÓN}

Ante la complejidad de los procesos de disciplinarización y desdibujamiento de las fronteras disciplinares en las ciencias sociales y ante las posturas inter - post - anti disciplinarias revisadas anteriormente, aparece como alternativa la transdisciplinariedad. El fundamento de esta es la emergencia de "campos de estudio" (Fuentes, 1998) que es un "movimiento hacia la superación de los límites entre especialidades cerradas y jerarquizadas y el establecimiento de un campo de discurso y prácticas sociales cuya legitimidad académica y social dependa cada vez más de la profundidad, extensión, pertinencia y solidez de las explicaciones que produzca y no del prestigio institucional acumulado" (Vasallo, 2001, p. 53) El de la comunicación es un campo que emerge de la relación orgánica entre las ciencias sociales y la comunicación. La comunicación en las sociedades contemporáneas está colocada en un lugar central, al grado que se le ha llegado a denominar "sociedad de la comunicación". Esto hace posible colocar a los estudios de comunicación como un campo de estudio que implica articular la experiencia y los recursos de diversas ramas y enfoques en una síntesis que confluya, en tanto lógica científica, para la producción de conocimiento pertinente y consistente y que responda a las necesidades sociales.

Esta forma de entender la transdisciplinariedad no supone romper con las formas institucionales que han tomado los estudios de comunicación sino la 
conquista del rigor teórico-metodológico y la ampliación y consolidación de los saberes que hasta ahora han estado fragmentados. En palabras de Jesús Martín Barbero (2002) "la propuesta latinoamericana es insertar la investigación de comunicación en el espacio de las ciencias sociales: la transdisciplinariedad... hay que pasar de la legitimidad teórica del campo a su legitimidad intelectual que es la posibilidad de que la comunicación sea un lugar estratégico desde el que pensar la sociedad”. (Martín Barbero, 2002, p. 211)

\section{LA COMUNICACIÓN: ESTUDIOS DE MEDIOS VS. ESTUDIOS DE LA CULTURA}

Al parecer los estudios de comunicación han asumido esta posición transdisciplinaria y esto ha implicado la construcción de objetos de investigación desde una nueva perspectiva. Es en el encuentro entre los tradicionales estudios de la comunicación centrados en los medios y sus efectos y desde una perspectiva cuantitativa de la investigación, con una nueva forma de entender la comunicación desde la cultura, que se plantea como objeto los procesos de producción social de sentido y se abre a metodologías de corte cualitativo, que se ha ido reconstruyendo el campo.

Este proceso ha supuesto una diversificación de objetos tal que al construir una mirada amplia nos encontramos con un campo caracterizado por una "investigación de la comunicación [que] ha tendido a ser más bien pluridisciplinaria (interacción existente entre dos o más disciplinas)... [una] constitución fragmentaria (y centrífuga) de marcos disciplinarios diversos... [con] la sociología como marco transdisciplinario para los estudios sobre la comunicación...” (Fuentes, 1998).

Concebir la comunicación como un proceso social más amplio, no sólo centrada en los medios, ha llevado a los estudios de comunicación por caminos nuevos. La construcción social de sentido es un proceso que no sólo se realiza en interacción con los medios masivos de comunicación sino que es un constitutivo básico de la interacción cotidiana y un proceso fundamental para la integración social. Esto ha sido planteado por algunos investigadores estadounidenses como James Carey (1989) quien señala que asumir la perspectiva cultural de la comunicación implica enfrentarnos a la pregunta ¿Dónde terminan las condiciones de vida y comienza la ciencia? ¿Hasta dónde la producción científica es una ideología más? Las estrategias para enfrentar tal paradoja, tanto en los estudios de comunicación en particular, como en las ciencias sociales en general han sido tres: 
- Concebir la comunicación como una ciencia del comportamiento cuyo objetivo es la elucidación de leyes.

- Concebir la comunicación como una ciencia formal cuyo objetivo es elucidar las estructuras.

- Concebir la comunicación como una ciencia de la cultura, cuyo objetivo es la elucidación del significado.

Optar por la tercera estrategia significa que el reto de los estudios de comunicación es tomar los significados que la gente construye en sus palabras y acciones y hacer estos significados, estos llamados acerca de la vida y la experiencia, explícitos y articuladores para que podamos juzgarlos claramente. La tarea es, entonces, construir una 'lectura' de la acción humana, interpretar las interpretaciones que los sujetos han construido sobre sus prácticas.

Tomar este camino implica asumir que la comunicación es algo sin esencia, sin cualidades universales, la comunicación simplemente constituye un conjunto de prácticas y reflexiones, históricamente variadas, sobre el mundo. "El significado es una forma de dar orden y coherencia al mundo para los propósitos humanos que se realizan al interior de un conjunto de relaciones sociales estructuradas y estructurantes" (Carey, 1989, p. 29)

Desde esta perspectiva el reto para la investigación de la comunicación de masas es "examinar los diversos mundos culturales en los cuales la gente existe simultáneamente, la tensión entre ellos, los patrones de ánimo y motivación distintivos de cada uno y la interpenetración entre ellos" (Carey, 1989, p. 60)

Ante una postura como la anteriormente expuesta, surgen voces que plantean que es necesario regresar al centro de los estudios de la comunicación las interacciones sociales que se han visto transformadas por las nuevas formas de comunicación masiva. Se hacen señalamientos respecto de que una perspectiva de la comunicación desde la cultura deja de lado la mirada sobre los medios de comunicación como instituciones sociales o como industrias culturales y se coloca en una postura relativista en la que toda interpretación posible es pertinente.

Aunque podemos reconocer que hay un intento por 'regresar' a las formas tradicionales de los estudios de medios, probablemente porque tener un campo de estudios bien delimitado permite construir una identidad académica fuerte, es evidente que el regreso como tal es, ahora, imposible. La amplia reflexión que se ha realizado en torno a la comunicación y la cultura ha llevado, a quienes están más interesados en la comunicación de masas, a plantear su trabajo desde una perspectiva más amplia, considerando los múltiples factores culturales e 
histórico - sociales que intervienen en los procesos de comunicación. Es difícil que, en estos tiempos, alguien se plantee estudiar, por ejemplo, los efectos de la comunicación sin considerar la complejidad de factores que intervienen.

En un esfuerzo por aclarar los 'malentendidos' que un planteamiento del estudio de la comunicación desde la cultura ha generado, Jesús Martín Barbero (2002) señala: "pensar la comunicación desde la cultura es hacer frente al pensamiento instrumental que ha dominado el campo desde su nacimiento, no es un abandono del campo sino su desterritorialización para diseñar un nuevo mapa de problemas en el que quepa la cuestión de los sujetos y las temporalidades sociales, es decir, la trama de la modernidad" Martín Barbero, 2002, p. 211)

Aún así, sigue abierto, para muchos, el debate respecto de cuáles son los objetos pertinentes a los estudios de comunicación y la necesidad de reconocer la diferencia entre estudios de la cultura y estudios de la comunicación.

\section{LOS OBJETOS Y METODOLOGÍAS PERTINENTES}

Los debates planteados en los apartados anteriores tanto el referido al estatus disciplinario de los estudios de comunicación como las visiones del estudio de la comunicación desde los medios o desde la cultura han establecido una gama muy amplia de posibles objetos y metodologías pertinentes para los estudios en este campo. En el trabajo concreto de investigación se han abordado múltiples objetos empíricos desde diversas posiciones teóricas y epistemológicas y siguiendo perspectivas metodológicas distintas.

Los estudios de comunicación en particular, como las ciencias sociales en general, avanzaron desde esfuerzos por construir un conocimiento que abarcara todos los aspectos implicados en la comprensión de lo social, hacia estudios más delimitados y particulares en términos históricos y geográficos. La multiplicación de objetos disparó los intereses de investigación fragmentándolos y, de algún modo, reduciéndolos.

A pesar de lo anterior se han ido construyendo, en los últimos años, modelos teóricos que pretenden establecer las bases de una comprensión amplia, compleja y transdisciplinar de la comunicación. El asunto está abierto al debate. Por ahora, propongo dos de estos modelos que pueden ayudar como puntos de partida para este debate. Son recuperaciones sintéticas de un pensamiento más amplio y complejo, planteado por cada uno de los autores que los han construido y que han sido expuestos ampliamente en sus publicaciones recientes. Es necesario enfatizar que son considerados sólo como punto de partida 
para ese debate mayor respecto de las perspectivas teóricas que pueden y deben acompañar los estudios de comunicación.

\section{LA PROPUESTA DE THOMPSON}

Thompson (1998) entiende la comunicación de masas como: "la producción institucionalizada y difusión generalizada de bienes simbólicos a través de la fijación y transmisión de información o contenido simbólico"

La comunicación de masas cuenta con una serie de características típicas:

- Implica ciertos medios de producción y difusión técnicos e institucionales. Es por este carácter institucional que algunos los denominan "industrias culturales" o "industrias mediáticas".

- Implica la explotación comercial de las innovaciones técnicas y la producción de formas simbólicas para el consumo.

- Instituye una ruptura estructurada entre la producción de formas simbólicas y su recepción.

- Extiende la disponibilidad de las formas simbólicas en el espacio y el tiempo

- Las formas simbólicas quedan a disposición de cualquiera que posea los medios técnicos, capacidades y recursos para adquirirlos, es decir, son formas simbólicas de circulación pública.

Para producir o recibir formas simbólicas a través de soportes técnicos se requieren habilidades y competencias específicas. Es necesario distinguir aquellas que se requieren para codificar la información o contenidos simbólicos y las que se requieren para decodificar el mensaje. Algunas pueden coincidir pero otras no. Thompson señala además: "Cuando los individuos codifican o descodifican mensajes, emplean no sólo las habilidades y competencias requeridas por los soportes técnicos, sino también varias formas de conocimiento y presuposiciones que comprenden parte de los recursos culturales que se dan durante los procesos de intercambio" (Thompson, 1998, p. 43).

$\mathrm{El}$ aspecto central de la comunicación de masas es que los medios técnicos modifican sustancialmente las formas de acción e interacción humana, los modos en que se producen, transmiten y reciben las formas simbólicas. Si consideramos, como Giddens (1995), que la estructuración de la sociedad (estructuración sistémica y estructuración social) está relativamente determinada por la interacción de los agentes sociales, podemos entonces reconocer que la modificación de las formas de interacción social producida por el desarrollo 
de los medios técnicos, es uno de los aspectos centrales de la comunicación de masas. A este asunto Thompson le llama "impacto interaccional" de los medios técnicos y reconoce cuatro dimensiones de este impacto:

- Los medios facilitan la interacción a través del espacio y del tiempo y producen una forma de interacción que Thompson denomina semi - interacción mediada.

- Los medios afectan la manera en que los individuos actúan para otros, en la medida en que los otros para quienes lo hacen pueden constituir un público extendido, disperso y distante. Se redefinen las formas de auto-representación y las regiones anteriores y posteriores de los espacios de interacción.

- Afectan la manera en que los individuos actúan en respuesta a otros, los medios de comunicación posibilitan formas de acción (acción concertada) y reacción que se extienden o comprimen en el tiempo y que rebasan las fronteras de los Estados-nación.

- Afectan las maneras en que los individuos actúan e interactúan en el proceso de recepción, es decir, afectan la organización social de las esferas de la vida cotidiana donde la actividad de recepción es una actividad rutinaria.

En su teoría de la comunicación de masas, Thompson plantea que el desarrollo de la comunicación masiva ha generado una reconstitución de los límites entre la vida pública y la privada. Los medios han posibilitado el carácter público (visibilidad) de sucesos o individuos tanto del campo público como del privado con independencia del lugar físico en el que se sitúen, es desde allí que se construyen las categorías de sucesos públicos mediados y sucesos privados mediados. Esta nueva condición de los sucesos cambia su naturaleza, la esfera pública ya no requiere ni de la co-presencia ni de una interacción dialógica.

Por otro lado, los sucesos mediados (públicos y privados) invaden el espacio privado en el proceso de recepción (ámbito doméstico o laboral privado) y modifican las interacciones de los individuos, sean receptores principales o secundarios. Se genera también una mayor accesibilidad y participación en la esfera pública, lo que dificulta la capacidad de controlar y limitar la información para quienes ejercen el poder.

Al revisar el desarrollo de los marcos institucionales para la comunicación de masas Thompson plantea que las instituciones de los medios deberían ocupar un espacio entre la operación desenfrenada de las fuerzas del mercado y el control directo del Estado. Para ello propone desarrollar un pluralismo 
regulado que implica la desconcentración de recursos en las industrias de los medios y la separación clara entre las instituciones de los medios y el ejercicio del poder estatal.

Desde estos planteamientos generales Thompson propone tres campos - objetos para el estudio de la comunicación:

- La producción y transmisión o difusión de las formas simbólicas que implica reconstruir las condiciones socio - históricas y los contextos de producción de las formas simbólicas, las reglas y convenciones, las relaciones e instituciones sociales y la distribución del poder, los recursos y las oportunidades en virtud de las cuales estos contextos forman campos diferenciados y socialmente estructurados. Este primer campo-objeto deberá abordarse a través del análisis socio - histórico y de la investigación etnográfica.

- La construcción del mensaje de los medios que tiene como propósito develar la estructura articulada de los objetos y expresiones significativas que circulan en los campos simbólicos, entendiéndolas como construcciones simbólicas complejas. Para abordar este campo - objeto Thompson propone el análisis formal o discursivo a través del análisis semiótico, el análisis conversacional, el análisis sintáctico, el análisis narrativo y el análisis argumentativo.

- La recepción o apropiación de los mensajes de los medios, es decir, la indagación respecto de cómo los mensajes son recibidos por los individuos o grupos de individuos, que se sitúan en circunstancias socio - históricas específicas y que emplean los recursos que tienen a su disposición para entender los mensajes recibidos y para incorporarlos en sus vidas diarias. Este tercer campo - objeto también se deberá abordar a través del análisis socio - histórico y la investigación etnográfica.

Thompson señala que "un enfoque comprehensivo para el estudio de la comunicación masiva requiere la capacidad de relacionar entre sí los resultados de estos diferentes análisis, demostrando cómo se alimentan e iluminan entre sí los diversos aspectos" (Thompson, 1998, p. 441).

\section{UNA PROPUESTA LATINOAMERICANA}

Desde América Latina es Jesús Martín Barbero quien ha hecho el esfuerzo más sistemático y consolidado por plantear un mapa que ayude a debatir la pertinencia de los objetos de investigación para la comunicación. 
Para Martín Barbero (2002) pensar la comunicación en América Latina es una tarea de envergadura antropológica pues lo que está en juego es la vida cotidiana de las mayorías que se ha transformado profundamente. Esta transformación se debe a que las masas en nuestro continente se han incorporado a la modernidad a través de los formatos y los géneros de las industrias culturales audiovisuales, especialmente la televisión. Esto lleva, necesariamente, a que los estudios de comunicación busquen abordar la relación comunicación/cultura sin totalizarla.

Para ello propone pensar las mediaciones comunicativas de la cultura con un nuevo mapa que logre dar cuenta de las relaciones constitutivas de la comunicación en la cultura y afronte el pensamiento único que legitima que la tecnología es el 'gran mediador' entre las audiencias y la realidad.

Desde el mapa que propone es necesario incorporar a los estudios de comunicación la transformación de las mediaciones socioculturales (tradicionales y nuevas) que introducen nuevos sentidos de los social y nuevos usos sociales de los medios. Esto implica "asumir el desafío político, técnico y expresivo...del espesor cultural que hoy contienen los procesos y los medios de comunicación... [y reconocer] el lento alumbramiento de nuevas esferas de lo público" (Martín Barbero, 2002, p. 226)

El mapa se articula sobre dos ejes, uno diacrónico que se constituye de la tensión entre las matrices culturales y los formatos industriales; y uno sincrónico que se constituye en la tensión entre las lógicas de producción y las competencias de recepción y consumo. Las relaciones posibles entre los polos de ambas tensiones se encuentran mediadas por diversos procesos. El mapa propuesto por el autor se podría visualizar de la siguiente forma:

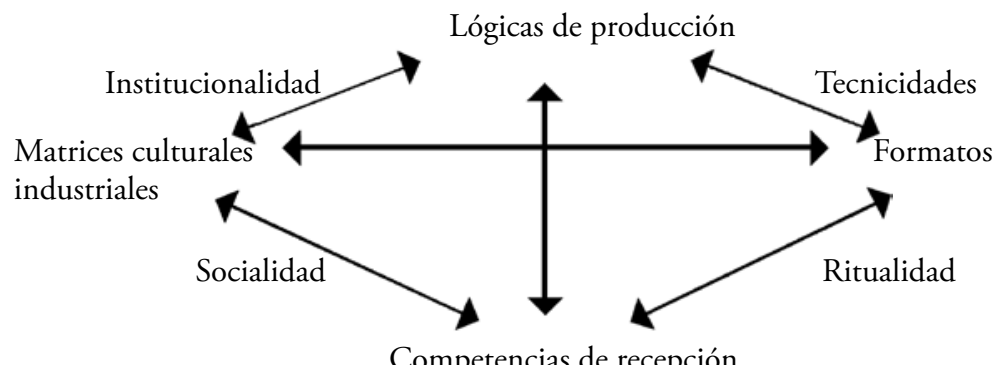

Competencias de recepción

La propuesta es que la investigación en comunicación se oriente al estudio de las mediaciones, en este caso, la socialidad, la ritualidad, la institucionalidad y las tecnicidades. 
La socialidad es entendida como "la trama de relaciones cotidianas que tejen los hombres al juntarse y en la que anclan los procesos primarios de interpretación y constitución de los sujetos y las identidades... lo que en la socialidad se afirma es la multiplicidad de modos y sentidos en que la colectividad se hace y se recrea, la polisemia de la interacción social" (Martín Barbero, 2002, pp. 227-228).

La ritualidad es "lo que en la comunicación hay de permanente reconstrucción del nexo simbólico...es lo que en el intercambio hay de forma y de ritmo...la ritualidad pone reglas al juego de la significación introduciendo el mínimo de gramaticalidad que hace posible expresar y compartir el sentido" (Martín Barbero, 2002, p. 228).

La institucionalidad "atraviesa la comunicación en dos órdenes contrapuestos: el que desde el Estado configura a los medios de comunicación como 'servicio público', y el que desde el mercado convierte la 'libertad de expresión' en libre comercio... mirada desde la institucionalidad la comunicación se convierte en cuestión de medios, esto es de producción de discursos públicos cuya hegemonía se haya hoy, paradójicamente, del lado de los intereses privados." (Martín Barbero, 2002, p. 230).

La tecnicidad "nombra entonces lo que en la sociedad no es sólo el orden del instrumento sino también de la sedimentación de saberes y la constitución de prácticas...la técnica es un organizador de prácticas...del diseño de nuevas prácticas...y competencia en el lenguaje." (Martín Barbero, 2002, p. 231).

El autor propone, además, cuatro mediaciones que es necesario trabajar para que el análisis y la crítica puedan vincularse creativamente a la producción: las estructuras sociales, las lógicas de producción, las dinámicas culturales y las gramáticas discursivas.

\section{Puntos de Convergencia}

Las propuestas para la elaboración de una Teoría de la Comunicación planteadas por los autores revisados coinciden en algunos aspectos que recupero muy brevemente:

- Los dos proponen comprender la comunicación de masas como una práctica cultural.

- Los autores coinciden en que los procesos de comunicación de masas no están en los medios sino en los procesos de interacción social entre los medios y las audiencias. 
- Los dos reconocen que los medios y su desarrollo en el siglo XX han modificado sustancialmente las formas de interacción social.

- Esta modificación de las formas de interacción social ha modificado también los límites y la comprensión que tenemos de la esfera pública.

- Los dos proponen un proceso interpretativo para comprender la comunicación de masas como práctica cultural.

Aunque algunos autores privilegian los estudios de recepción, es necesario comprender que siguen una tendencia muy marcada en los estudios de comunicación recientes. La controversia entre los académicos, en este nivel, es amplia y de larga data. Es necesario señalar que es imprescindible construir objetos que aborden la relación entre las tres instancias señaladas por Thompson y que son uno de los núcleos de la controversia: los procesos de producción y difusión, la construcción o contenidos de los mensajes y los procesos de recepción o apropiación. Si logramos construir objetos que se sostengan en la tensión o relación entre estas instancias lograremos tomar una posición epistemológica que evite reducir la comunicación a los mensajes o que, finalmente, evite centrarse únicamente en la recepción sin considerar las condiciones socio-históricas en las que se posibilita la comunicación.

Adicionalmente, si asumimos que son los agentes, en sus interacciones cotidianas, los que constituyen las estructuras sociales y al mismo tiempo están determinados por éstas, es necesario construir objetos que aborden la relación entre ambas.

Sobre estas propuestas teóricas y las posibles posiciones que pueden adoptar los agentes del campo en relación a ellas, el debate continúa abierto.

\section{LA ARTICULACIÓN POR CONSTRUIR}

El proceso de institucionalización del campo de la comunicación en México, se originó en función de la aparición de los medios electrónicos masivos, es decir, la radio y la televisión. Aunque la comunicación masiva no surge con el advenimiento de la radio y la televisión, pues la prensa de finales del siglo XIX ya tenía las características de una comunicación de masas, el oficio del periodismo no consideraba la necesidad de una formación profesional específica.

Con la aparición de los medios electrónicos se comienza a constituir un campo profesional específico y la necesidad de tener sujetos formados en las universidades para desempeñar las tareas que la producción de comunicación requiere. En México en la década de 1960, surgen las primeras licenciaturas en periodismo y comunicación en las universidades, con la intención de formar 
profesionales de la comunicación. Es allí donde surge la comunicación como un ámbito universitario específico. El proceso de institucionalización de este ámbito de la formación profesional experimenta un crecimiento acelerado y consistente durante casi tres décadas. Se multiplican los programas de formación profesional con énfasis diversos, con múltiples proyectos sociales a la base. El proceso de institucionalización se consolida pues se conforman departamentos de comunicación, al interior de las universidades, y asociaciones que tienen como centro la enseñanza de la comunicación.

Aunque a nivel internacional los estudios de comunicación aparecieron desde la década de 1920, en México es en la década de 1970 cuando da inicio el proceso de investigación académica de la comunicación. Sobre el desarrollo institucional de los programas de formación profesional se constituyen grupos y sujetos universitarios (inicialmente profesores) interesados en realizar investigación sistemática sobre la comunicación. Algunos sujetos salen a formarse en posgrados del extranjero y otros se forman en los nacientes posgrados en comunicación, cuyo interés central es formar investigadores de la comunicación. Hay claros indicadores del desarrollo y consolidación de este subcampo de la investigación de la comunicación como el surgimiento de publicaciones especializadas y la conformación de la Asociación Mexicana de Investigadores de la Comunicación (AMIC), además de la constitución de programas de Maestría y más recientemente, de doctorados en comunicación.

Desde esta mirada el campo de la comunicación alberga en su interior, al menos, tres esferas o subcampos que se constituyeron y han operado de forma desarticulada: la esfera profesional de la comunicación, la esfera de formación profesional de comunicadores y la esfera de la investigación de la comunicación. Adicionalmente la esfera profesional tiene, en su interior, diversas áreas como son la producción de comunicación, la comunicación organizacional, la publicidad o la investigación comercial de la comunicación, entre otras.

Esta desarticulación, que responde a condiciones históricas de la emergencia del campo e incluso a historias de relación personal entre los propios agentes del campo, es quizá la mayor debilidad a la que nos enfrentamos. Ante esta situación de desarticulación Sánchez Ruiz (2002) hace un llamado a "generar las pertinencias mutuas y las correspondencias entre los subcampos" (Sánchez, 2002, p. 30) y plantea algunas articulaciones posibles:

- Los investigadores "académicos" interactúan con los sujetos sociales del subcampo de la comunicación profesional a través de la investigación empírica. 
- Los resultados de la investigación comercial o aplicada pueden ser recuperados por la investigación académica para generar argumentaciones explicativas e interpretativas más amplias.

- Los hallazgos y teorías formuladas desde la investigación académica enriquecen y contextualizan el trabajo de la investigación comercial o aplicada y el trabajo de los profesionales de la comunicación.

- Los docentes enriquecen su trabajo educativo con los hallazgos de la investigación académica y comercial, incorporando los hallazgos de estas a los cursos.

- Los investigadores académicos sirven de consultores expertos a quienes diseñan las políticas de comunicación, a los que toman decisiones en el ámbito profesional y a grupos de la sociedad civil.

Con esto queda abierto el debate respecto de si realmente es un camino viable y deseable la articulación propuesta y respecto de las estrategias que, quienes participamos del campo y creemos necesaria la articulación, podemos echar a andar para lograr avanzar en este camino.

\section{Conclusiones}

El campo de los estudios de comunicación enfrenta, actualmente, retos muy importantes entre los que podemos señalar al menos cuatro:

- Asumir la transdisciplinariedad como lugar de construcción de objetos de estudio.

- Reconocer la importancia de la relación comunicación/cultura sin totalizarla o, a partir de ello, fragmentar más el campo de estudios de la comunicación.

- Desarrollar líneas, metodologías y perspectivas teóricas pertinentes.

- Avanzar hacia la articulación entre investigación, enseñanza y profesión.

Enfrentar estos retos de un modo constructivo, que lleve al campo de los estudios de comunicación a ganar mayor legitimidad científica y social, implica necesariamente pasar por un debate amplio de los asuntos aquí planteados.

Debatir a través de un diálogo abierto, reconociendo que las posibilidades son múltiples, asumiendo los procesos socio - históricos tanto del desarrollo de las ciencias sociales y la constitución del campo, como de los procesos de construcción social de sentido y trabajando en una posible articulación y enriquecimiento mutuo de los subcampos. 
La agenda, por supuesto más amplia y compleja que los puntos expuestos en este artículo, ha sido planteada muchas veces en el pasado y desde perspectivas muy diversas. La reiterada reflexión sobre estas cuestiones no es más que un síntoma de la "insatisfacción generalizada con el estado actual del campo y la urgencia de repensar sus fundamentos y de reorientar el ejercicio de sus prácticas" (Vasallo, 2001, p. 49)

Para avanzar en el diálogo - debate se pueden realizar acciones, relativamente sencillas y al alcance de la mano, como reconocer los posibles núcleos de debate, leer con inteligencia y profundidad el trabajo de los colegas, abrir espacios de discusión centrados en algunos de los temas específicos, involucrar a los diversos agentes del campo: académicos (investigadores y profesores) y profesionales en estos espacios de discusión, construir intencionadamente relaciones con agentes de otros subcampos para enriquecer el propio trabajo, poner en relación más abierta las asociaciones nacionales y latinoamericanas centradas en la enseñanza con las que agrupan el trabajo de los investigadores.

Seguramente el diálogo y debate de estas cuestiones no nos llevará a construir acuerdos generalizados y asumidos por todos, no es ésta la pretensión ni el propósito final, sino a fortalecer al propio campo al asumir las tensiones que lo constituyen, asumir la propia posición y tomar un papel activo en él, reconociendo la innegable diversidad de posibilidades que lo conforman. Es, en última instancia, convivir con la incertidumbre como característica fundacional de todo proceso social y de la propia condición humana.

BiBLIOGRAFÍA

Carey W. (1989). Communication as Culture. Essays on Media and Society. New York \& London: Routledge

Fuentes R. (1998). La emergencia de un campo académico: continuidad utópica y estructuración científica de la investigación de la comunicación en México. Guadalajara: ITESO/Universidad de Guadalajara.

Jensen, K. (1995). The Social Semiotics of Mass Communication. London: Sage.

Jensen, K. (2002). A Handbook of Media and Communication Research. Qualitative and Quantitative Methodologies. London \& New York: Routledge.

Katz, P., Liebes \& Orloff. (2003). Canonic Texts in Media Research. Cambridge UK: Polity Press.

Martín, J. (2002). Itinerarios de la investigación, Oficio de Cartógrafo. Travesías latinoamericanas de la comunicación en la cultura. Santiago de Chile: Fondo de Cultura Económica. 
Ortiz, R. (1999). Ciencias sociales, globalización y paradigmas, en Reguillo y Fuentes (coords.). Pensar las ciencias sociales hoy: Reflexiones desde la cultura. Guadalajara: ITESO.

Sánchez, E. E. (2002). La investigación latinoamericana de la comunicación y su entorno social: notas para una agenda, Diálogos de la Comunicación, No. 64, Lima: FELAFACS.

Thompson, J. B. (1998). Ideología y Cultura Moderna. Teoría crítica social en la era de la comunicación de masas. México: UAM-UX.

Vasallo, M. I. (2001). Reflexiones sobre el estatuto disciplinario del campo de la comunicación, en Lopes y Fuentes (coords.). Comunicación: campo y objeto de estudio. Perspectivas reflexivas latinoamericanas. Guadalajara: ITESO/ U.A.A.I UdeCol./ UdeG.

Wallerstein, I. (1996). Le futur des sciences sociales. Recuperado de http://www.uqac. uquebec.ca/zone30/Classiques des sciences sociales/index.html

Wallerstein, I. (Coord.) (1996). Abrir las Ciencias Sociales. Informe de la comisión Gulbenkian para la reestructuración de las ciencias sociales. México: Siglo XXI / Centro de Investigaciones Interdisciplinarias en Ciencias Sociales y Humanidades UNAM

Wallerstein, I. (1999). Impensar las ciencias sociales: limites de los paradigmas decimonónicos (Parte VI). México: Siglo XXI.

Wallerstein, I. (2004). The uncertainties of knowledge. Phildalephia: Temple University Press. pp. 7-58. 